\title{
Effects of Atrial Natriuretic Factor on Cyclic Guanosine Monophosphate and Cyclic Adenosine Monophosphate Accumulation in Microdissected Nephron Segments from Rats
}

\author{
Hiroshi Nonoguchi, Mark A. Knepper, and Vincent C. Manganiello \\ National Heart, Lung and Blood Institute, National Institutes of Health, Bethesda, Maryland 20892
}

\begin{abstract}
Atrial natriuretic factor (ANF) (1 $\mu \mathrm{M})$ markedly increased cyclic guanosine monophosphate (cGMP) content in microdissected glomeruli (35-fold) and in microdissected inner medullary collecting ducts (IMCD) (20-fold). ANF caused little or no increase in cGMP content in other nephron segments. The threshold concentration for increased cGMP accumulation by ANF was 0.1$1 \mathrm{nM}$ in IMCD, which is in the range reported for rat plasma. Sodium nitroprusside (1 mM), which selectively stimulates soluble guanylate cyclase, increased cGMP content in glomeruli but not in IMCD.

ANF did not alter cAMP accumulation in the absence or presence of vasopressin (AVP) or parathyroid hormone (PTH) in outer and inner medullary tubule suspensions, or in microdissected proximal convoluted tubules (PCT), medullary thick ascending limbs (MAL) or IMCD.

These data are compatible with the hypothesis that cGMP is a second messenger for a physiologic action of ANF in the inner medullary collecting duct. ANF apparently activates membrane-bound guanylate cyclase in this segment.
\end{abstract}

\section{Introduction}

Atrial natriuretic factors (ANFs), ${ }^{1}$ peptides released from mammalian cardiac atria, exhibit potent natriuretic, diuretic and smooth muscle relaxant properties (1-4). They have been purified and sequenced (5-9) and have been characterized with regard to their physiological effects in intact animals (1-4). Relatively little is known, however, about the renal mechanism of ANF action or about the precise target sites for ANF along the nephron. While effects of ANF on glomerular filtration may account for a substantial part of their natriuretic response $(5$, 10), effects on renal tubular $\mathrm{NaCl}$ and water transport may also play an important role in the overall renal response $(11,12)$.

The actions of many hormones are mediated by cyclic nucleotides. Earlier studies have shown that ANF increases urinary excretion of cGMP in the dog (13) and increases cGMP accu-

Address reprint requests to Dr. Knepper, Room 6N307, Bldg. 10, National Institutes of Health, Bethesda, MD 20892.

Received for publication 31 March 1986 and in revised form 14 July 1986

1. Abbreviations used in this paper: ANF, atrial natriuretic factor; AVP, arginine vasopressin; CAL, cortical thick ascending limb; CCD, cortical collecting duct; DT, distal tubule, IBMX, 3-isobutyl-1-methylxanthine; IMCD; inner medullary collecting duct; MAL, medullary thick ascending limb; OMCD, outer medullary collecting duct; PCT, proximal convoluted tubule; PST, proximal straight tubule; PTH, parathyroid hormone; TDL, thin descending limb.

The Journal of Clinical Investigation, Inc.

Volume 79, February 1987, 500-507 mulation in tubule suspensions from dogs (14), in rat glomeruli $(15,16)$, and in cultured rat renal papillary tissue $(17,18)$. These and other studies in vascular smooth muscle $(19,20)$ raise the possibility that CGMP may be a second messenger in ANF action. Therefore, in the present study, we used microdissected nephron segments to define precisely those segments of the rat nephron in which ANF increases cGMP content.

In addition, some of the actions of ANF could be due to effects on intracellular cAMP levels. Consequently, we have also examined effects of ANF on peptide hormone-induced changes in the cAMP content of microdissected nephron segments.

\section{Methods}

Materials. Synthetic atrial natriuretic factors (ANF [6-33], ANF [8-33] and ANF [13-28]) and synthetic bovine parathyroid hormone (PTH) (134) were purchased from Peninsula Laboratories (Belmont, CA). Except where specifically noted, the 28-amino acid peptide ANF (6-33) (also called Ser-Leu-Arg-Arg-atriopeptin-III or cardionatrin I) was used in all experiments. This has been identified as the chief circulating form of ANF in the rat (9). Prostaglandin $\mathrm{E}_{2}\left(\mathrm{PGE}_{2}\right)$ was obtained from PL Biochemicals (Piscataway, NJ). 3-isobutyl-1-methylxanthine (IBMX), arginine vasopressin (AVP), (-)epinephrine (+)bitartrate, DL-propranolol hydrochloride, sodium nitroprusside, L-glutamine, L-alanine and collagenase (type 1) were obtained from Sigma Chemical Co. (St. Louis, MO). Bovine serum albumin (BSA) was obtained from Armour Pharmaceuticals (Kankakee, IL).

Preparation of tubule suspensions. Preliminary experiments were done in tubule suspensions from rabbits and rats. Male and female rabbits (New Zealand White, 1-3 kg, Small Animal Breeding Facility, National Institutes of Health [NIH]) were injected with pentobarbital $(40 \mathrm{mg} / \mathrm{kg}$ body wt i.v.). Furosemide $(10 \mathrm{mg}$ ) was injected intravenously $15 \mathrm{~min}$ before injection of pentobarbital to eliminate medullary osmolality gradients. The kidneys were separately perfused via renal arteries with 10 $\mathrm{ml}$ of ice-cold physiological saline (solution 1), which contained (in $\mathrm{mM}$ ): $\mathrm{NaCl}, 117 ; \mathrm{KCl}, 5.0 ; \mathrm{NaHCO}_{3}, 25 ; \mathrm{NaH}_{2} \mathrm{PO}_{4}, 1.0 ; \mathrm{MgSO}_{4}, 1.2 ; \mathrm{CaCl}_{2}$, 2.0; glucose, $5.5 ; \mathrm{L}$-alanine, 6.0 ; and $\mathrm{BSA}, 1 \mathrm{mg} / \mathrm{ml}$; and was equilibrated with $95 \% \mathrm{O}_{2}-5 \% \mathrm{CO}_{2}$. The kidneys were then perfused with $\sim 15 \mathrm{ml}$ of ice-cold solution 1 containing collagenase (type 1 ), $1 \mathrm{mg} / \mathrm{ml}$. Inner medulla or outer medulla were carefully dissected, minced, and incubated in solution 1 containing collagenase at $37^{\circ} \mathrm{C}$ for $1 \mathrm{~h}$ (for inner medullary tubule suspensions) or $\mathbf{4 5} \mathrm{min}$ (for outer medullary tubule suspensions). Suspensions were then taken up into and expelled from a 5-ml pipette five times and filtered through three layers of gauze. The suspensions were centrifuged (200-400 $g$ for 3-4 min). The pellets were washed four or five times by resuspending and centrifuging the pellets in solution 2 . Solution 2 contained (in $\mathrm{mM}$ ): $\mathrm{NaCl}, 135 ; \mathrm{KCl}, 5.0 ; \mathrm{NaH}_{2} \mathrm{PO}_{4}, 1.0$; $\mathrm{MgSO}_{4}, 1.2 ; \mathrm{CaCl}_{2}, 2.0 ;$ glucose, 5.5 ; L-alanine, $6.0 ; \mathrm{N}$-2-hydroxyethylpiperazine- $N^{\prime}$-2-ethanesulfonic acid (Hepes), 10.0 and $\mathrm{pH}$ was adjusted to 7.4 by $\mathrm{NaOH}$. Finally, tubules were suspended in $200-400 \mu \mathrm{l}$ of solution 2 and placed on ice until use.

In preparing tubule suspensions from rats (pathogen-free male Sprague-Dawley rats weighing $200-350 \mathrm{~g}$ from NIH Small Animal Breeding Facility), the animals were decapitated and kidneys were perfused via the aorta. Suspensions were then prepared as described above for rabbits. 
Preparation of isolated nephron segments from rats. Pathogen-free male Sprague-Dawley rats (100-200 g) were decapitated and the left kidney was perfused via the abdominal aorta with $10 \mathrm{ml}$ of solution 1 . The abdominal aorta was ligated just above and below the left renal artery. The left kidney was then perfused with $10 \mathrm{ml}$ of solution 1 containing collagenase and removed. Kidney slices were cut along a corticomedullary axis and were incubated with solution 1 containing collagenase for $30 \mathrm{~min}$ at $37^{\circ} \mathrm{C}$ under aeration with $95 \% \mathrm{O}_{2}-5 \% \mathrm{CO}_{2}$. After incubation, the slices were washed four or five times with solution 2.

Microdissection of tubules was performed using needles under a stereomicroscope at $17^{\circ} \mathrm{C}$ in solution 2 containing $0.05 \%$ BSA.

The segments that were dissected are illustrated in Fig. 1. Glomeruli, mid-proximal convoluted tubules (PCT) and distal tubules (DT) were microdissected from the cortical labyrinth. The DT is a heterogeneous segment that includes distal convoluted tubules, connecting tubules and initial collecting ducts. Early proximal straight tubules (early PST or S2) were dissected from the upper part of the medullary rays in the cortex. Cortical thick ascending limbs (CAL) and cortical collecting ducts (CCD) were also dissected from medullary rays in the cortex. Late proximal straight tubules (late PST or S-3) were from the outer stripe of the outer medulla. Thin descending limbs of Henle's loop from the outer medulla (TDL) were identified by their attachment to late PST. Medullary thick ascending limbs (MAL) and outer medullary collecting ducts (OMCD) were taken from the inner stripe of the outer medulla. Inner medullary collecting ducts (IMCD) were taken from the inner medulla. Because the upper third of IMCD is morphologically and functionally different from middle and lower thirds $(21,22)$, we took IMCD only from the lower two-thirds (mainly from middle third) of the inner medulla. Thin limbs of Henle's loop from the inner medulla (TL[IM]) included both thin descending and ascending limbs, which could not be differentiated with our current dissection techniques. (Because vasa recta are similar to thin limbs in size and appearance, we cannot rule out the presence of vasa recta among the thin limbs dissected from inner medulla.)

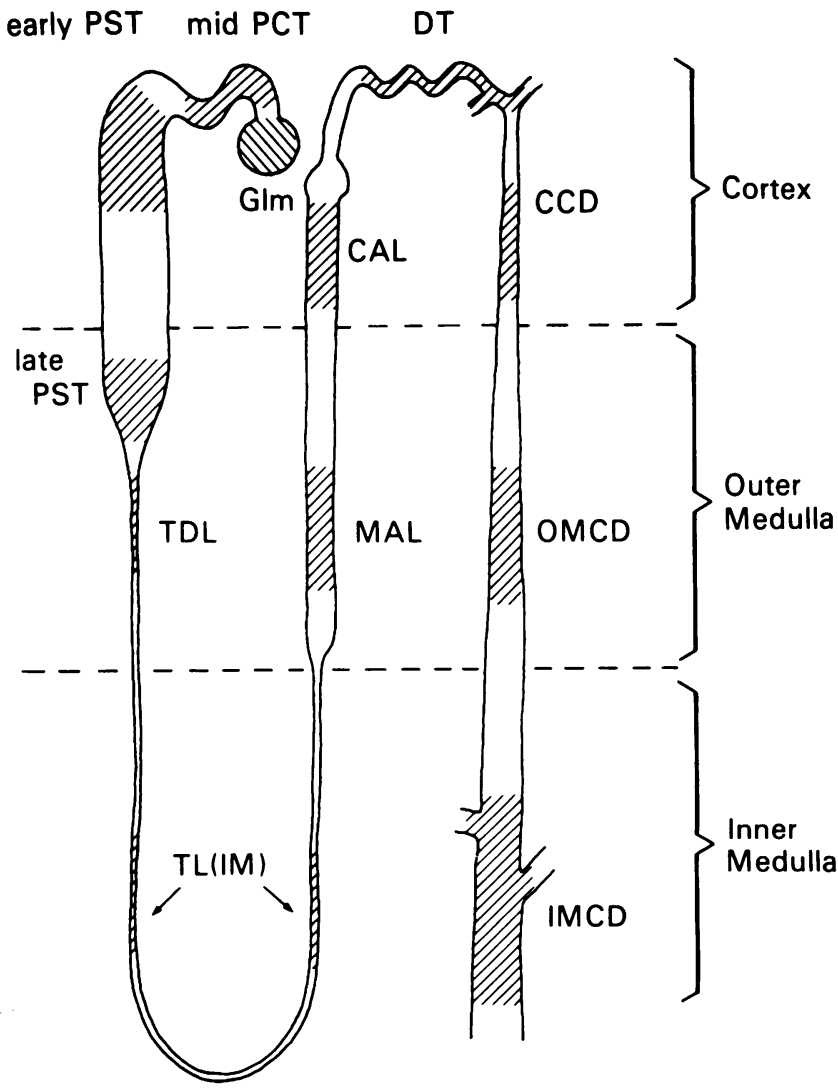

Figure 1. Schematic representation of microdissected nephron segments. See abbreviations in the text.
After microdissection, tubules were transferred to another dish filled with solution 2 containing $0.05 \%$ BSA. Tubular length was measured by ocular micrometer. Mean lengths of tubules used for a single determination of cGMP content were as follows: mid-PCT, $30.6(\mathrm{~mm})$; early PST, 22.4; late PST, 29.8; TDL, 23.7; TL(IM), 30.6; MAL, 29.3; CAL, 23.4; DT, 10.4; CCD, 15.7; OMCD, 12.2; IMCD, 9.8. Usually three to five glomeruli per sample were incubated in experiments in which glomerular CGMP accumulation was measured. Segments of 1-3 mm length from PCT, MAL, or IMCD were incubated in experiments in which cAMP content was measured.

\section{Incubation of tubule suspensions and nephron segments}

Measurement of cyclic nucleotide content. In general, slight modifications of the procedures described by Torikai and Kurokawa (23) were used.

Tubule suspensions ( $10 \mu \mathrm{l}$ containing 6-50 $\mu \mathrm{g}$ protein for inner medullary suspensions, $200-400 \mu \mathrm{g}$ protein for outer medullary suspensions) were added to a $1.5-\mathrm{ml}$ plastic centrifuge tube containing $10 \mu \mathrm{l}$ of solution 2 with $1 \mathrm{mM}$ IBMX at $4^{\circ} \mathrm{C}$.

Microdissected nephron segments were transferred with $2 \mu \mathrm{l}$ of dissection solution using a siliconized glass pipette into 1.5 -ml plastic centrifuge tubes containing $18 \mu \mathrm{l}$ of solution 2 with $0.56 \mathrm{mM}$ IBMX. For experiments with proximal convoluted and straight tubules, the composition of solution 2 was changed to maintain viability of the segments; L-glutamine $(1 \mathrm{mM})$ and sodium acetate $(3 \mathrm{mM})$ were added and $\mathrm{NaCl}$ was reduced accordingly to maintain osmolality.

The remainder of the incubation protocol was the same for tubule suspensions and microdissected nephron segments. Suspensions or microdissected tubules were incubated for $10 \mathrm{~min}$ at $37^{\circ} \mathrm{C}$ (at 50 oscillations/ min in a shaking bath) before addition of $20 \mu \mathrm{l}$ of solution 2 containing indicated concentrations of ANF or sodium nitroprusside. In experiments in which cGMP content was measured, after incubation for $3 \mathrm{~min}$, the reaction was stopped by addition of $50 \mu 1$ of $10 \%$ TCA. In experiments in which cAMP was measured, after incubation for $3 \mathrm{~min}$ in the presence or absence of ANF, $20 \mu \mathrm{l}$ of solution 2 with or without indicated compounds (AVP, PTH, prostaglandin $\mathrm{E}_{2}\left[\mathrm{PGE}_{2}\right]$, and epinephrine plus propranolol) was added. After an additional $2 \mathrm{~min}$, the reaction was stopped by addition of $70 \mu \mathrm{l}$ of $10 \%$ TCA. Each tube was centrifuged for $5 \mathrm{~min}$ at $8,700 \mathrm{~g}$ using a microcentrifuge (Beckman Instruments, Inc., Palo Alto, CA) and supernatant (for measurement of cGMP, $80 \mu \mathrm{l}$ from a total $90 \mu \mathrm{l}$; for cAMP, $115 \mu \mathrm{l}$ from a total $130 \mu \mathrm{l}$ ) was stored at $-20^{\circ} \mathrm{C}$ for $<5 \mathrm{~d}$ before assay of CGMP and cAMP. In each experiment, blank samples (containing medium and TCA without tubules) were also processed.

Frozen samples (including blank samples) were thawed at room temperature and the TCA was removed by extraction four times with 0.75 or $1.0 \mathrm{ml}$ of water-saturated ether. After evaporation of ether, the remaining aqueous phase was dried using a speed vacuum concentrator (SVC $200 \mathrm{H}$, Savant Instruments Inc., Hicksville, NY) and $100 \mu \mathrm{l}$ of 50 $\mathrm{mM}$ sodium acetate buffer ( $\mathrm{pH} 6.2$ ) was added to each sample. For each concentration point on the standard curve, appropriate CGMP and CAMP standards were added to blank samples carried through the extraction procedure. Samples of unknowns and cGMP and cAMP standards were then assayed for cGMP and/or cAMP (radioimmunoassay kits, New England Nuclear, Boston, MA).

In tubule suspensions, the amounts of cyclic nucleotides were sufficiently high so that extracts from individual incubations could be diluted, and CGMP and cAMP content could be measured in duplicate in the same sample. With microdissected nephron segments, the amount of cGMP or cAMP in the samples was such that only a single determination of either CGMP or cAMP could be made per sample. The amount of cGMP in individual samples was generally too small to detect in the absence of IBMX. (In contrast, cAMP content was generally so high in microdissected tubules that some experiments could be done in the absence of IBMX.) In microdissected nephron segments, there was a linear relationship between cGMP content and either the number of glomeruli incubated without and with ANF (Fig. $2 A$ ) or the length of IMCD segments incubated without and with ANF (Fig. 2 B). To some tissue 

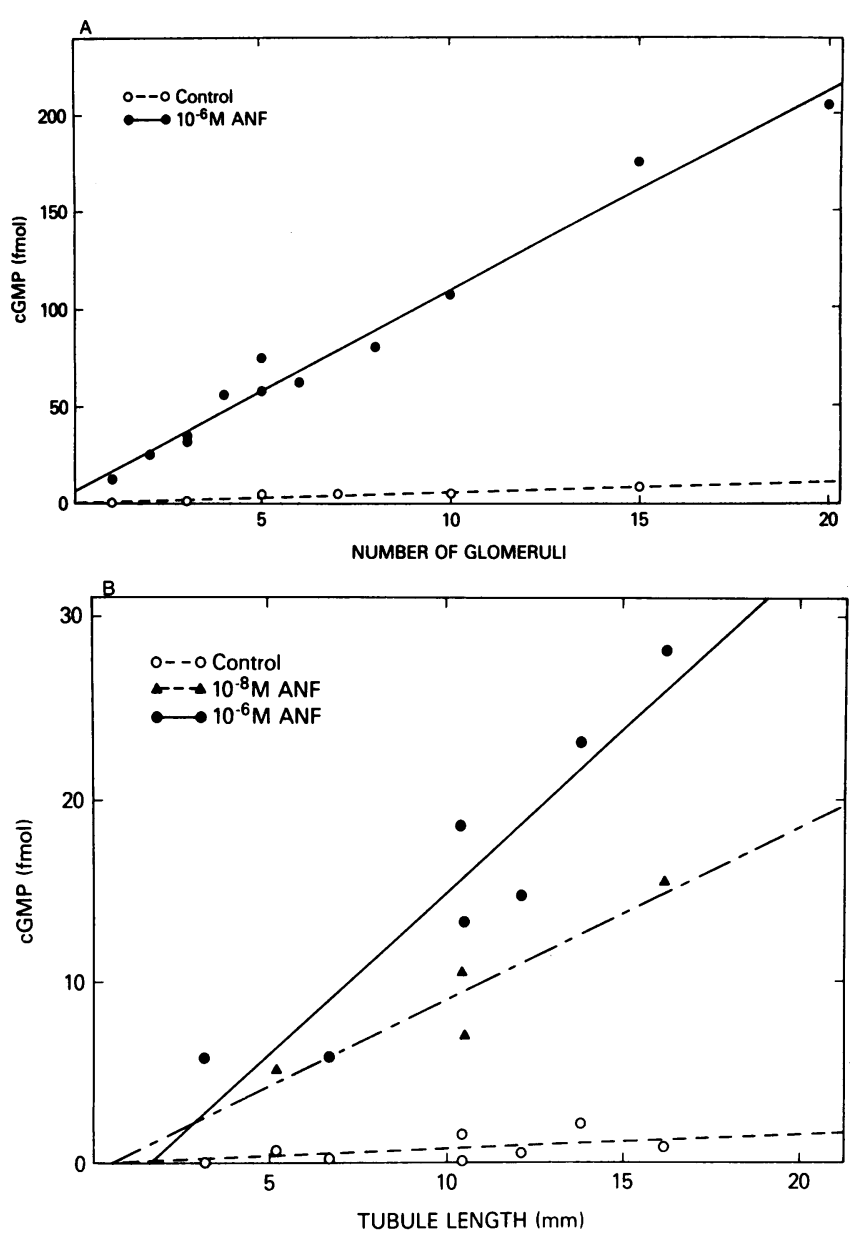

Figure 2. Relationship between number of glomeruli (A) or IMCD length (B) and CGMP accumulation. (A) Number of glomeruli. The indicated number of glomeruli were incubated with $1 \mu \mathrm{M}$ ANF (solid circle) and vehicle (open circle) for $3 \mathrm{~min}$ at $37^{\circ} \mathrm{C}$ and cGMP content was measured as described in Methods. $r=0.990$ (solid circle) and 0.918 (open circle). (B) IMCD tubular length. IMCD segments of indicated length were incubated with vehicle (open circle), $10 \mathrm{nM}$ ANF (solid triangle) and $1 \mu \mathrm{M}$ ANF (solid circle) and cGMP content was measured as described in Methods. Regression lines are $y=-0.09$ $+0.09 \mathrm{X}(r=0.501)$ for control, $y=-0.48+0.95 \mathrm{X}(r=0.930)$ for $10 \mathrm{nM}$ ANF, and $y=-2.95+1.79 \mathrm{X}(r=0.931)$ for $1 \mu \mathrm{M}$ ANF.

extracts, $1-50$ fmol authentic cGMP or 5-50 fmol cAMP were added. In these experiments, $94.9 \pm 2.6 \%$ (mean \pm SE, $n=15$ ) of added cGMP and $99.0 \pm 5.2 \%(n=11)$ of added cAMP were recovered. Since recovery of added cyclic nucleotide was essentially complete, and since addition of labeled tracer to samples would reduce accuracy of radioimmunoassay, internal cGMP and cAMP standards were not added to samples to monitor recovery.

Protein determinations. Protein content of tubule suspensions were measured with Coomassie Brilliant Blue G-250 (Bio-Rad Laboratories, Richmond, CA) using gamma-globulin as standard.

Statistical evaluation. Statistical analysis was performed using Student's $t$ test or analysis of variance with multiple comparisons by Dunnett's method. A $P$ value $<0.05$ was considered significant. Results are expressed as mean \pm SEM.

\section{Results}

Effects of ANF in tubule suspensions from rabbits and rats. Preliminary experiments were done with tubule suspensions. The

time course of the effect of ANF on cGMP accumulation in inner medullary tubule suspensions from rabbits and rats is shown in Fig. $3 \mathrm{~A}$. In the presence of $1 \mu \mathrm{M}$ ANF and $0.5 \mathrm{mM}$ IBMX, maximal accumulation was obtained within $3 \mathrm{~min}$ in suspensions from rabbits or $1 \mathrm{~min}$ in suspensions from rats. Based on these results, in subsequent studies, tubule suspensions and nephron segments were incubated with agents for 3 min before measurement of cGMP content.

Fig. $3 B$ shows the relationship between ANF concentration and CGMP accumulation in inner medullary tubule suspensions. ANF $(1 \mu \mathrm{M})$ increased cGMP content sevenfold in inner medullary tubule suspensions from rabbits and fourfold in suspensions from rats (Fig. $3 B$ ). The threshold concentration for an increase in CGMP was between 1 and $10 \mathrm{nM}$ in both rabbits and rats. Sodium nitroprusside $(1 \mathrm{mM})$ also increased cGMP content of inner medullary tubule suspensions from rabbits and rats (Fig. $3 A$ and $B$ ), but had no effect on cAMP content (data not shown).

To test the requirement for IBMX, tubule suspensions from rabbit inner medulla were incubated in the absence of IBMX. $1 \mu \mathrm{M}$ ANF increased cGMP content fivefold (from $1.4 \pm 0.2 \mathrm{fmol} /$ $\mu \mathrm{g}$ protein $(n=6)$ to $7.3 \pm 1.0 \mathrm{fmol} / \mu \mathrm{g}$ protein $(n=6))$. The mean ANF-stimulated cGMP content was $\sim 20 \%$ of that in the presence of IBMX (compare Fig. $3 \mathrm{~B}$ ). Thus, we used IBMX in all subsequent studies except where specifically indicated.

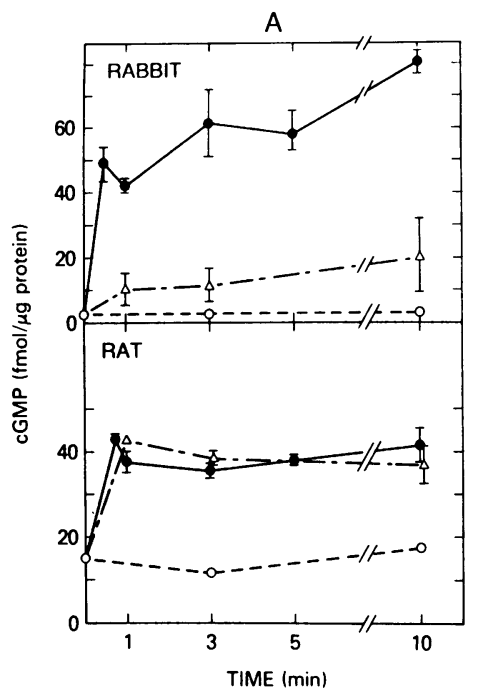

B

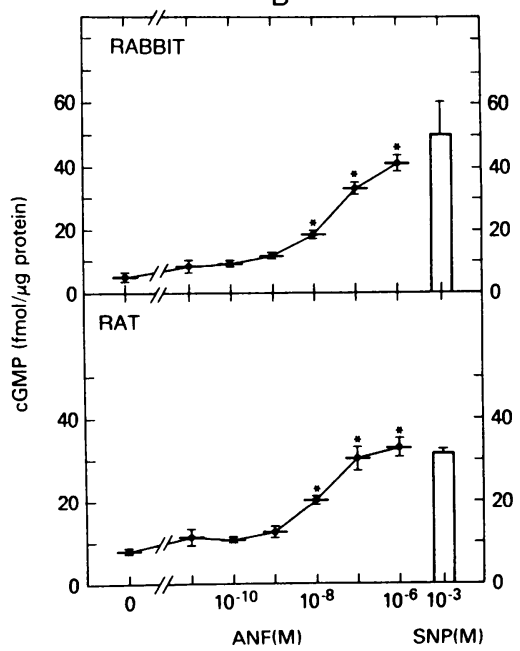

Figure 3. Time course (A) and concentration dependency (B) of ANF effect on cGMP accumulation in inner medullary tubule suspensions from rabbits and rats. (A) Tubule suspensions were incubated with $1 \mu \mathrm{M}$ ANF (solid circle), $1 \mathrm{mM}$ sodium nitroprusside (SNP) (open triangle) or vehicle (open circle) as indicated, and cGMP content was measured as described in Methods. Values are mean \pm SEM of 2-4 measurements from 2-4 rabbits or rats. (B) Tubule suspensions were incubated with indicated concentrations of ANF, $1 \mathrm{mM} \mathrm{SNP}$ or vehicle for $3 \mathrm{~min}$. Values represent mean \pm SEM of 3-10 measurements from 2-5 rabbits or rats. ${ }^{*} P<$ 0.05 vs. vehicle. 
Two forms of ANF, ANF (6-33) and ANF (8-33), produced virtually identical effects on CGMP accumulation in inner medullary tubule suspensions from rabbits and rats (data not shown). The 16-amino acid fragment ANF (13-28) had no effect. For all remaining studies in this report, we used ANF (6-33).

In tubule suspensions from rabbit outer medulla, $1 \mu \mathrm{M}$ ANF increased CGMP content fivefold (from $0.62 \pm 0.05(n=3)$ to $2.94 \pm 0.53(n=3) \mathrm{fmol} / \mu \mathrm{g}$ protein) and the threshold for a response was between 1 and $10 \mathrm{nM}$. The response was $\sim 14$-fold lower in outer medullary tubule suspensions $(2.94 \mathrm{fmol} / \mu \mathrm{g}$ protein) than in rabbit inner medullary tubule suspensions (41.3 fmol/ $\mu \mathrm{g}$ protein).

ANF did not affect cAMP content in tubule suspensions from the inner medulla of rabbits or rats or from the outer medulla of rabbits (data not shown).

cGMP accumulation in microdissected glomeruli and nephron segments from rats. Incubation of microdissected glomeruli for 3 min with $1 \mu \mathrm{M}$ ANF increased CGMP accumulation 35fold (from a control level of $0.48 \pm 0.02(n=7)$ to $16.6 \pm 2.2(n$ $=8) \mathrm{fmol} /$ glomerulus). The minimal concentration of ANF ("threshold dose") required to produce a measurable increase in CGMP accumulation was between $10 \mathrm{nM}$ and $100 \mathrm{nM}$ (Fig. 4). Sodium nitroprusside $(1 \mathrm{mM})$ increased cGMP content of microdissected glomeruli 13-fold (from a control level of $0.46 \pm 0.10(n=6)$ to $5.83 \pm 0.32(n=12) \mathrm{fmol} /$ glomerulus $)$.

The effects of $1 \mu \mathrm{M}$ ANF on cGMP accumulation in microdissected collecting duct segments are shown in Fig. 5. ANF increased cGMP in inner medullary collecting ducts (IMCD) 20-fold (from a control level of $0.07 \pm 0.02[n=8]$ to $1.50 \pm 0.14$ [ $n=7] \mathrm{fmol} / \mathrm{mm}$ ), and slightly increased cGMP accumulation in CCD. There was no significant effect of ANF in OMCD. Incubation of IMCD with $10 \mathrm{nM}$ ANF increased cGMP content 13-fold, about half that produced by $1 \mu \mathrm{M}$ ANF (Fig. 6). $1 \mathrm{nM}$ ANF also increased cGMP content three- to fivefold (Fig. 6). Sodium nitroprusside ( $1 \mathrm{mM})$ did not significantly increase cGMP content of IMCD and produced very small increments $(\sim 0.1 \mathrm{fmol} / \mathrm{mm})$ in CCD and CAL (data not shown).

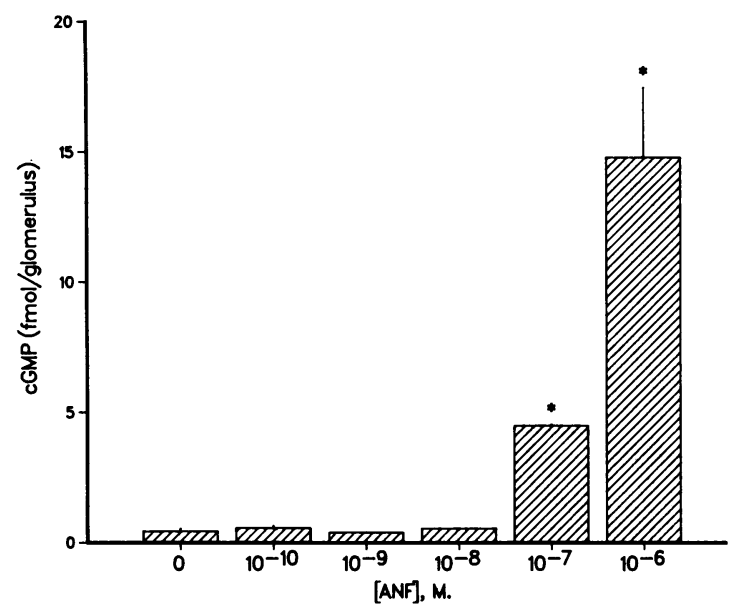

Figure 4. Concentration dependency of ANF effect on cGMP accumulation in microdissected glomeruli. Glomeruli were incubated with indicated concentrations of ANF or vehicle for 3 min and CGMP content was measured as described in Methods. Values are mean \pm SEM of 3-4 determinations. ${ }^{*} P<0.05$ for difference between control and ANF treated.

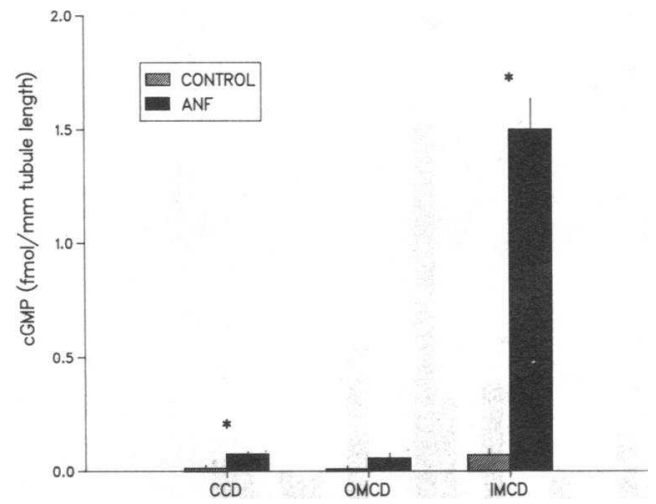

Figure 5. Effects of ANF on CGMP accumulation in collecting ducts. Collecting ducts were incubated without or with $1 \mu \mathrm{M}$ ANF for $3 \mathrm{~min}$, and CGMP content was measured as described in Methods. Values are mean \pm SEM of 4-8 determinations. ${ }^{*} P<0.05$.

Fig. 7 shows CGMP accumulation in nephron segments other than collecting ducts incubated in the absence or presence of 1 $\mu \mathrm{M}$ ANF. (Note that the vertical axis scale is expanded relative to that for collecting duct segments [compare Fig. 5]. Thus, all responses in noncollecting duct segments were small relative to the response in the IMCD.) Basal cGMP content was highest in thin limbs from the inner medulla (TL [IM]) $(0.11 \mathrm{fmol} / \mathrm{mm})$ and relatively high in CAL and distal tubules (DT) $(0.04$, and $0.03 \mathrm{fmol} / \mathrm{mm}$, respectively). In other segments, basal cGMP content was very low $(<0.02 \mathrm{fmol} / \mathrm{mm})$. ANF did not signifcantly alter CGMP accumulation in late PST, CAL, and DT and produced small, but significant increases in CGMP content $(<0.1$ $\mathrm{fmol} / \mathrm{mm}$ ) in mid-PCT and early PST. Incubation with $1 \mu \mathrm{M}$ ANF also produced small increments $(<0.3 \mathrm{fmol} / \mathrm{mm})$ in cGMP

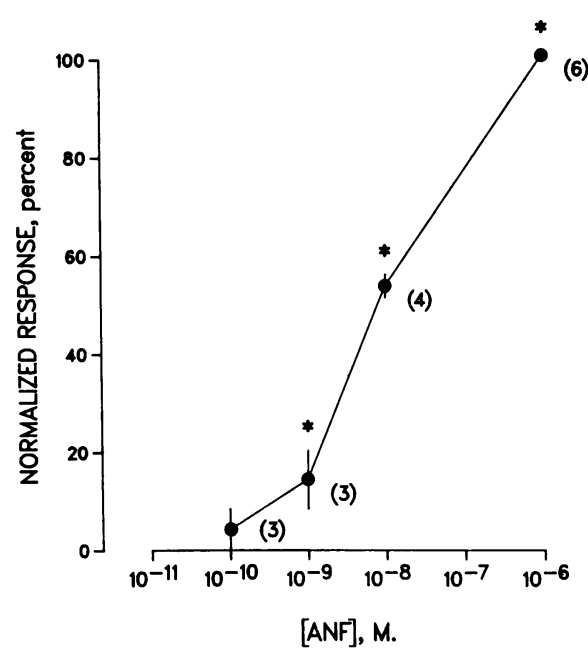

Figure 6. Concentration dependency of ANF-induced increases in CGMP accumulation in microdissected IMCD. IMCD segments were incubated with indicated concentrations of ANF and vehicle for 3 min, and CGMP content was measured as described in Methods. Normalized values represent the differences in CGMP content of segments incubated with the indicated concentrations of ANF minus vehicle, divided by the value of cGMP content at $1 \mu \mathrm{M}$ ANF. Values are means \pm SEM. $(n)$ indicates number of rats. ${ }^{*} P<0.05$ vs. zero. 


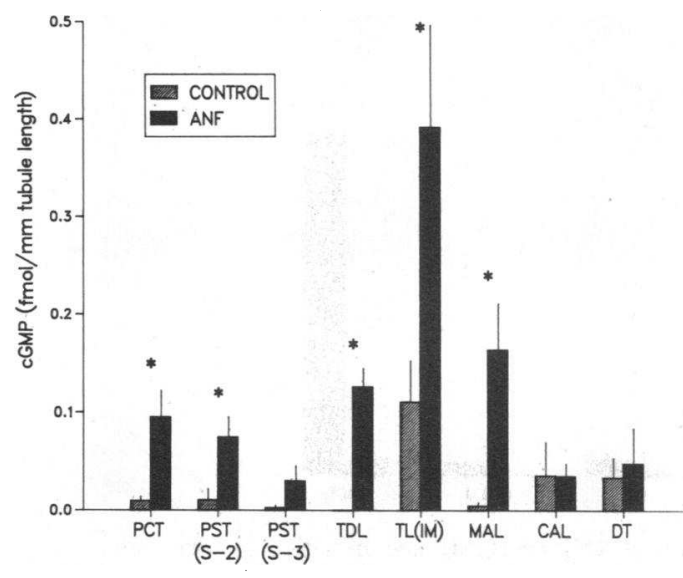

Figure 7. Effects of ANF on cGMP accumulation in microdissected nephron segments. Nephron segments were incubated with $1 \mu \mathrm{M}$ ANF. CGMP content was measured as described in Methods. From each portion of the nephron, segments of approximately equal length were incubated without or with $1 \mu \mathrm{M}$ ANF. Values represent mean \pm SEM (3-7 determinations). ${ }^{*} P<0.05$. Note that scale is different from Fig. 5 .

content in thin descending limbs from the outer medulla (TDL), TL (IM), and MAL. In MAL segments, a lower concentration of ANF (10 nM) also increased cGMP content (from a control level of $0.01 \mathrm{fmol} / \mathrm{mm}$ to $0.12 \pm 0.03 \mathrm{fmol} / \mathrm{mm}$ ).

Effects of ANF on AVP- or PTH-induced accumulation of cAMP. In these experiments, tubule suspensions or microdissected nephron segments were exposed to ANF for $3 \mathrm{~min}$ and then were incubated with AVP or PTH for additional 2 min before measurement of cAMP content.

In rabbit inner medullary tubule suspensions, $1 \mu \mathrm{M}$ ANF had no significant effect on cAMP accumulation in the presence of $1 \mathrm{nM}$ AVP [without ANF or AVP: $40.8 \pm 2.3(n=6) \mathrm{fmol} /$ $\mu$ g protein; AVP alone: $75.0 \pm 8.6(n=7)$; ANF plus AVP: $74.7 \pm 11.9(n=7)]$. Similar observations were made in outer medullary tubule suspensions from rabbits (data not shown).

In microdissected IMCD, ANF $(1 \mu \mathrm{M})$ had no significant effect on cAMP accumulation in response to $0.1 \mathrm{nM}$ AVP (Fig. 8). To provide a positive control, we tested the effect of epinephrine $(0.5 \mu \mathrm{M})$ plus propranolol $(0.5 \mu \mathrm{M})$ which is known to inhibit AVP-dependent cAMP accumulation in cortical and

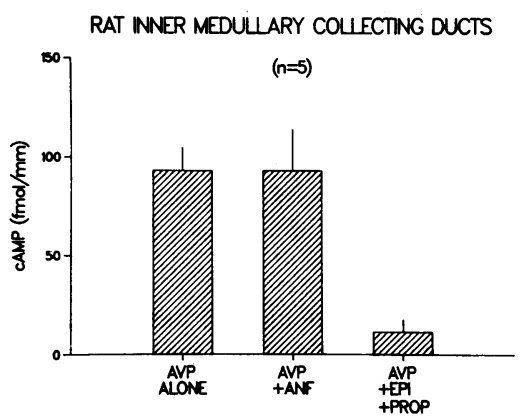

Figure 8. Effect of ANF $(1 \mu \mathrm{M})$ or epinephrine $(0.5 \mu \mathrm{M})$ plus propranolol $(0.5 \mu \mathrm{M})$ on AVP-induced increases in cAMP accumulation in IMCD. In five different experiments, IMCD were incubated with or without ANF $(1 \mu \mathrm{M})$ for $3 \mathrm{~min}$ and for an additional 2 min with AVP(0.1 nM): A third

group of tubules was incubated with AVP $(0.1 \mathrm{nM})$ plus epinephrine $(0.5 \mu \mathrm{M})$ plus propranolol $(0.5 \mu \mathrm{M})$. Values for basal cAMP content were $3.1 \pm 1.9 \mathrm{fmol} / \mathrm{mm}$ (mean $\pm \mathrm{SEM}, n=5$ ). Each bar shows mean value for five rats (2-4 determinations were done per rat.) outer medullary collecting ducts (24). In IMCD, this combination inhibited the AVP-stimulated increase in cAMP accumulation by $\sim 90 \%$ (Fig. 8). (0.5 $\mu \mathrm{M}$ clonidine [an $\alpha_{2}$-adrenergic agonist] also blocked the AVP-stimulated increase in cAMP accumulation. Furthermore, $5 \mu \mathrm{M}$ yohimbine [an $\alpha_{2}$-adrenergic receptor blocker] prevented the effect of epinephrine or clonidine to inhibit cAMP accumulation. These data suggest that the inhibition of AVP-stimulated cAMP accumulation by epinephrine in the IMCD is an $\alpha_{2}$-adrenergic effect.) In other experiments, ANF was found to have no effect on cAMP accumulation in IMCD in the absence of AVP or on AVP-stimulated cAMP accumulation in the absence of IBMX (data not shown).

In MAL, a target site for AVP action, $1 \mu \mathrm{M}$ ANF did not inhibit cAMP accumulation in the absence or presence of $1 \mathrm{nM}$ (Fig. 9) or $10 \mathrm{nM}$ AVP $(43.7 \pm 3.4 \mathrm{fmol} / \mathrm{mm}$ with $10 \mathrm{nM}$ AVP $[n=5$ ], $38.5 \pm 4.5$ with $1 \mu \mathrm{M}$ ANF plus $10 \mathrm{nM}$ AVP $[n=5]$ ). In contrast, $1 \mu \mathrm{M} \mathrm{PGE}{ }_{2}$, a known inhibitor of AVP-induced increases of cAMP accumulation in the MAL, blocked the increase in cAMP content induced by AVP by $68 \%$ (Fig. 9) (In the presence of $10 \mathrm{nM}$ AVP, PGE $_{2}$ inhibited the stimulation by $82 \%$ ).

Since PTH is known to stimulate adenylate cyclase in the proximal tubule, the effects of ANF on PTH-induced increases in cAMP content were studied in microdissected PCT. In four experiments using 6.7 or $67 \mathrm{nM}$ PTH, $1 \mu \mathrm{M}$ ANF showed no effect on PTH-induced increase of cAMP content. Similar results

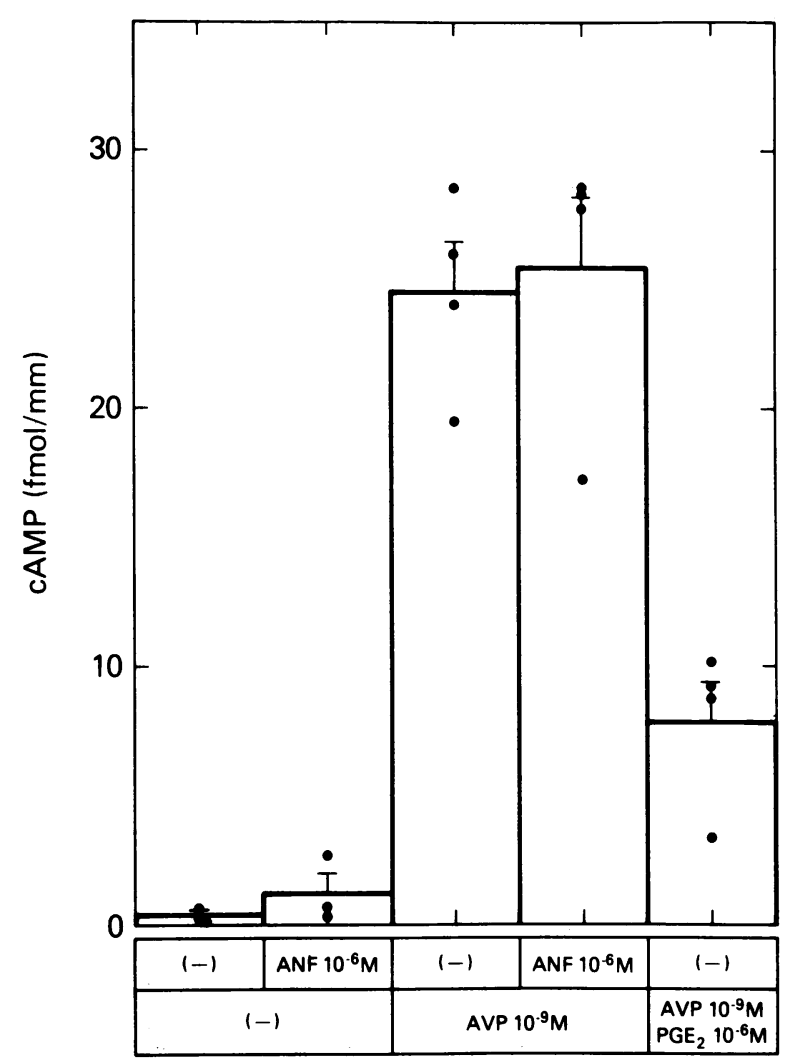

Figure 9. Effects of ANF $(1 \mu \mathrm{M})$ on cAMP accumulation in the absence and presence of AVP in MAL. Nephron segments were incubated with the indicated concentrations of effectors as described in Fig. 8. In this experiment, all samples contained $0.03 \%$ ethanol. Each point represents one sample; the horizontal bar represents mean \pm SEM. 
have been recently reported by others (25). In contrast, epinephrine $(0.5 \mu \mathrm{M})$ plus propranolol $(0.5 \mu \mathrm{M})$ inhibited the PTHinduced increase of cAMP content by $64 \%$ [from $36.4 \pm 4.2$ ( $n$ $=5)$ to $13.1 \pm 1.0 \mathrm{fmol} / \mathrm{mm}(n=3)]$.

In PCT incubated without IBMX, PTH $(0.67 \mu \mathrm{M})$ increased cAMP content threefold. ANF $(1 \mu \mathrm{M})$ did not alter PTH-stimulated cAMP accumulation in the absence of IBMX (data not shown).

\section{Discussion}

ANF effects on cGMP accumulation in nephron segments. Although little is known regarding the mechanism of action of ANF at the cellular level, cGMP is considered to be a likely intracellular or second messenger of $\operatorname{ANF}(19,20)$. ANF has been demonstrated to increase membrane-bound, but not soluble, guanylate cyclase activity in broken cell preparations from smooth muscle $(20)$ and kidney $(15,19)$, and is known to stimulate cGMP accumulation in glomeruli (14-16), tubule suspensions from thick ascending limbs and collecting ducts from dogs (14), primary cultures of inner medullary collecting ducts (17, 18) and renal epithelial cell lines (26). The chief objective of the present studies was to determine precisely, using microdissected rat nephron segments, those segments of the nephron in which ANF influences cGMP metabolism.

Our results show that among renal tubular segments, the inner medullary collecting duct exhibits the largest increase in cGMP content in response to ANF (Figs. 5 and 7). From the point of view of percentage change from basal cGMP levels, the response in inner medullary collecting ducts was comparable to that seen in glomeruli (compare Fig. 4). Although small increases in cGMP content were observed in proximal convoluted tubules, proximal straight tubules, thin limbs of Henle's loop, medullary thick ascending limbs of Henle's loop, and cortical collecting ducts, the magnitude of the response in inner medullary collecting ducts was so much greater that we chose to focus our further attention chiefly on this segment.

Experiments investigating the relationship between ANF concentration and CGMP accumulation in the IMCD revealed that the threshold ANF concentration for a response was between 0.1 and $1 \mathrm{nM}$ (Fig. 6). Determinations of ANF levels in circulating blood plasma in rats have disclosed basal ANF concentrations in a similar range $(0.02$ to $3 \mathrm{nM})(27-30)$, with higher values following increases in extracellular fluid volume $(28,30)$ or after vasopressin administration (27). Thus, the dose-response relationship in IMCD is compatible with a physiological action of ANF to increase CGMP in the IMCD. The concentration of ANF required to stimulate cGMP accumulation in microdissected IMCD is not only within the range of reported ambient ANF plasma concentrations, but is also within the reported range of ANF concentrations which produce at least two other presumed physiological effects of ANF, i.e., half-maximal smooth muscle relaxation ( $<1 \mathrm{nM}$ ANF) (31) and inhibition of aldosterone secretion $(<2 \mathrm{nM})(32,33)$.

In view of the failure of sodium nitroprusside, a known stimulator of soluble (i.e., cytosolic) guanylate cyclase, to increase cGMP accumulation in microdissected IMCD, we presume that ANF increases cGMP accumulation in the IMCD chiefly by activating membrane-bound guanylate cyclase. (In contrast to microdissected IMCD, inner medullary tubule suspensions responded to sodium nitroprusside with an increase in cGMP ac- cumulation. Stimulation of cGMP accumulation by sodium nitroprusside in tubule suspensions presumably is due to the presence of tissue elements other than IMCD, which contain soluble guanylate cyclase, most likely either vasa recta or thin limbs of Henle's loop.) Although no conclusions can be drawn regarding the roles of membrane-bound and soluble guanylate cyclase in cellular function, activation of membrane-bound guanylate cyclase has generally been found to be receptor mediated and limited to only several peptides including $\operatorname{ANF}(15,18,19,34)$ and Escherichia coli heat-stable enterotoxin (35-37). Activation of soluble guanylate cyclase on the other hand may be a secondary event in the action of a number of effectors, related perhaps to changes in $\mathrm{Ca}^{2+}$ mobilization, redox state of the cell, or arachidonate and prostaglandin metabolism $(34,38)$. These considerations further emphasize the importance of the selective nature of the response of IMCD to ANF, implying that regulation of cGMP in IMCD may occur primarily via receptor-mediated activation of a membrane-associated guanylate cyclase.

Whereas effects of ANF on renal hemodynamics and glomerular filtration are well documented (1-4), a renal tubular site of action is not yet generally accepted. However, the demonstration in the present study that ANF, at concentrations measured in systemic blood plasma from rats, can increase cGMP production by IMCD adds to the rapidly accruing evidence that ANF may have a physiologic action on the inner medullary collecting duct. Previously, from collecting duct microcatheterization studies, Sonnenberg et al. (12) suggested that ANF-induced natriuresis results largely from effects of ANF in the inner medulla associated with decreased $\mathrm{NaCl}$ absorption in the IMCD. In addition, Davis et al. (39) reported that ANF greatly attenuates axial solute gradients in inner medulla, consistent with a local site of action in the inner medulla. High affinity ANF binding sites have been demonstrated not only in glomeruli, but also in the inner medulla, probably localized to the IMCD $(15,40,41)$. Furthermore, the demonstration by Zeidel et al. (42) that ANF inhibits ouabain-sensitive oxygen consumption in cells isolated from IMCD also points to the IMCD as a likely site of ANF action.

ANF effects on cGMP accumulation in glomeruli. In confirmation of prior studies (14-16), we found that ANF causes a large increase in CGMP accumulation in glomeruli. In contrast to IMCD, glomeruli did not exhibit an increase in cGMP content in response to ANF at concentrations reported in systemic blood plasma in rats. The threshold concentration for an increase in cGMP accumulation by glomeruli was in the range of 10 to 100 nM (Fig. 4). Despite this observation, we would be reluctant to conclude that ANF does not have a physiological action at the glomerular level for several reasons: (a) Physiological actions of ANF may be mediated by second messengers other than cGMP in glomeruli. (b) Glomeruli are structurally complex with at least four cell types that could in principle respond to ANF (viz. mesangial cells, endothelial cells, visceral epithelial cells, and parietal epithelial cells). If the ANF-responsive cells are embedded within the structure of the glomerulus, higher than physiological ANF concentrations may be required in the incubation medium to allow enough ANF to penetrate to the responsive cell type to elicit a physiological response. (In contrast, normal blood perfusion of the glomerulus would distribute ANF and other circulating peptides throughout the structure of the glomerulus.) (c) Physiological actions of ANF on glomerular function may be mediated by effects on afferent and efferent arterioles that were not generally included with our microdissected glomeruli. 
Absence of ANF effect on cAMP accumulation. We could not demonstrate an effect of ANF on cAMP accumulation in any of several preparations studied including: (a) microdissected rat proximal convoluted tubules in the presence or absence of parathyroid hormone; $(b)$ microdissected medullary thick ascending limbs in the presence or absence of arginine vasopressin; (c) microdissected inner medullary collecting ducts in the presence or absence of arginine vasopressin; $(d)$ inner medullary tubule suspensions from rabbits in the presence of arginine vasopressin; and ( $e$ ) outer medullary tubule suspensions from rabbits in the presence of arginine vasopressin. In these studies, the phosphodiesterase inhibitor IBMX was included in the incubation mixture to maximize the cAMP response. Additional experiments were done in the absence of IBMX to examine the possibility that ANF affects cAMP metabolism through effects on phosphodiesterase activity. ANF again had no effect on cAMP accumulation in microdissected AVP-stimulated IMCD, or in microdissected PTH-stimulated PCT. Extensive efforts to undercover an effect of ANF on CAMP metabolism thus failed to demonstrate such an action. Umemura et al. (25) also found no effect of ANF on hormone-stimulated cAMP accumulation in PCT, thick ascending limbs, CCD, and OMCD. In contrast, Werness et al. (43) reported a small $(\sim 15 \%)$ inhibition of AVPstimulated cAMP accumulation by ANF in cortical collecting ducts. Appel and Dunn (17) have also reported that ANF does not alter CAMP accumulation in cultured IMCD cells.

Inhibition of AVP- and PTH-induced accumulation of cAMP by $\mathrm{PGE}_{2}$ or $\alpha_{2}$-adrenergic stimulation in proximal convoluted tubules, medullary thick ascending limbs, and inner medullary collecting ducts indicates that the inhibitory regulatory components of the adenylate cyclase system are functionally present and coupled to the catalytic unit of adenylate cyclase. Consequently, the failure of ANF to inhibit cAMP accumulation in these segments could not be due to the absence of a normally functioning inhibitory subunit.

$\alpha_{2}$-Adrenergic effect on hormone-stimulated cAMP accumulation. We have also demonstrated evidence for $\alpha_{2}$-adrenergic regulation of AVP responsiveness in IMCD. Incubation of IMCD with epinephrine plus propranolol (to prevent activation of $\beta$ adrenergic receptors) almost completely inhibited AVP-induced accumulation of cAMP. Umemura et al. $(24,44)$ have also reported $\alpha_{2}$-adrenergic regulation of cAMP accumulation in isolated proximal convoluted tubules, $\mathrm{CCD}, \mathrm{OMCD}$, and glomeruli. Additional studies are needed to characterize more fully, with appropriate analogues and blockers, both the biochemical properties as well as the physiological significance of $\alpha_{2}$-adrenergic regulation of CAMP accumulation in IMCD.

Conclusion. In summary, our studies of microdissected nephron segments have revealed that, in terms of cGMP production and accumulation, the IMCD is the major renal tubular site of action of ANF. The increase in cGMP accumulation (in percent change) was comparable to that seen in glomeruli and increases in cGMP could be elicited by physiological (i.e., normal circulating) concentrations of ANF. In contrast, ANF had no demonstrable effects on cAMP metabolism in the nephron segments studied. We conclude that the IMCD is a likely site of ANF action on renal tubular $\mathrm{NaCl}$ and water transport. Studies using isolated perfused tubules should therefore be useful in determining whether ANF and CGMP have direct regulatory roles in IMCD transport.

\section{Acknowledgments}

We thank Drs. Maurice Burg and Martha Vaughan for their critical reading of the manuscript. We also thank Dr. John Pisano, who died 26 March 1985, for his advice and encouragement during the early stages of the work.

\section{References}

1. Ballermann, B. J., and B. M. Brenner. 1985. Biologically active atrial peptides. J. Clin. Invest. 76:2041-2048.

2. Cantin, M., and J. Genest. 1985. The heart and atrial natriuretic factor. Endocrine Rev. 6:107-127.

3. Laragh, J. H. 1985. Atrial natriuretic hormone, the renin-aldosterone axis, and blood pressure-electrolyte homeostasis. N. Engl. J. Med. 313:1330-1340.

4. Maack, T., D. N. Marion, M. J. F. Camargo, H. D. Kleinert, J. H. Laragh, E. D. Vaughan, Jr., and S. A. Atlas. 1984. Effect of auriculin (atrial natriuretic factor) on blood pressure, renal function, and the reninaldosterone system in dogs. Am. J. Med. 77:1069-1075.

5. Atlas, S. A., H. D. Kleinert, M. J. Camargo, A. Januszewicz, J. E. Sealey, J. H. Laragh, J. W. Schilling, J. A. Lewicki, L. K. Johnson, and T. Maack. 1984. Purification, sequencing and synthesis of natriuretic and vasoactive rat atrial peptide. Nature (Lond.). 309:717-719.

6. Currie, M. G., D. M. Geller, B. R. Cole, N. R. Siegel, K. M. Fox, S. P. Adams, S. R. Eubanks, G. R. Galluppi, and P. Needleman. 1984. Purification and sequence analysis of bioactive atrial peptides (Atriopeptins). Science (Wash. DC). 223:67-69.

7. Flynn, T. G., M. L. deBold, and A. J. deBold. 1983. The amino acid sequence of an atrial peptide with potent diuretic and natriuretic properties. Biochem. Biophys. Res. Commun. 117:859-865.

8. Kangawa, K., and H. Matsuo. 1984. Purification and complete amino acid sequence of $\alpha$-human atrial natriuretic polypeptide ( $\alpha$-hANP). Biochem. Biophys. Res. Commun. 118:131-139.

9. Schwartz, D., D. M. Geller, P. T. Manning, N. R. Siegel, K. F. Fox, C. E. Smith, and P. Needleman. 1985. Ser-Leu-Arg-Arg-Atriopeptin 3: The major circulating form of atrial peptide. Science (Wash. DC). 229:397-400.

10. Huang, C. L., J. Lewicki, L. K. Johnson, and M. G. Cogan. 1985. Renal mechanism of action of rat atrial natriuretic factor. J. Clin. Invest. 75:769-773.

11. Briggs, J. P., B. Steipe, G. Schubert, and J. Schnermann. 1982. Micropuncture studies of the renal effects of atrial natriuretic substance. Pflügers Arch. 395:271-276.

12. Sonnenberg, H., W. A. Cupples, A. J. deBold, and A. T. Veress. 1982. Intrarenal localization of the natriuretic effect of cardiac atrial extract. Can. J. Physiol. Pharmacol. 60:1149-1152.

13. Hamet, P., J. Tremblay, S. C. Pang, F. Carrier, G. Thibault, J. Gutkowska, M. Cantin, and J. Genest. 1984. Effect of native and synthetic atrial natriuretic factor on cyclic GMP. Biochem. Biophys. Res. Commun. 123:515-527.

14. Tremblay, J., R. Gerzer, P. Vinay, S. C. Pang, R. Beliveau, and P. Hamet. 1985. The increase of CGMP by atrial natriuretic factor correlates with the distribution of particulate guanylate cyclase. FEBS (Fed. Eur. Biochem. Soc.) Lett. 181:17-22.

15. Ballermann, B. J., R. L. Hoover, M. J. Karnovsky, and B. M. Brenner. 1985. Physiologic regulation of atrial natriuretic peptide receptors in rat renal glomeruli. J. Clin. Invest. 76:2049-2056.

16. Stokes, T. J., Jr., C. L. McConkey, Jr., and K. J. Martin. 1986. Atriopeptin 3 increases CGMP in glomeruli but not in proximal tubules of dog kidney. Am. J. Physiol. 250:F27-F31.

17. Appel, R. G., and M. J. Dunn. 1985. Effect of synthetic natriuretic factor on cGMP synthesis in rat renal papillary collecting tubule cells. Clin. Res. 33:618a. (Abstr.)

18. Ishikawa, S., T. Saito, K. Okada, T. Kuzuya, K. Kangawa, and H. Matsuo. 1985. Atrial natriuretic factor increases cyclic GMP and 
inhibits cyclic AMP in rat renal papillary collecting tubule cells in culture. Biochem. Biophys. Res. Commun. 130:1147-1153.

19. Waldman, S. A., R. M. Rapoport, and F. Murad. 1984. Atrial natriuretic factor selectively activates particulate guanylate cyclase and elevates cyclic GMP in rat tissues. J. Biol. Chem. 259:14332-14334.

20. Winquist, R. J., E. Faison, S. A. Waldman, K. Schwartz, F. Murad, and R. M. Rapoport. 1984. Atrial natriuretic factor elicits an endotheliumindependent relaxation and activates particulate cyclase in vascular smooth muscle. Proc. Natl. Acad. Sci. USA. 81:7661-7664.

21. Knepper, M. A., and J. M. Sands. 1987. Urea permeability of inner medullary collecting duct and papillary surface epithelium. J. Clin. Invest. 79:138-147.

22. Madsen, K. M., and C. C. Tisher. 1986. Structural-functional relationship along the distal nephron. Am. J. Physiol. 250:F1-F15.

23. Torikai, S., and K. Kurokawa. 1983. Effect of $\mathrm{PGE}_{2}$ on vasopressin-dependent cell cAMP in isolated single nephron segments. $\mathrm{Am}$. J. Physiol. 245:F58-F66.

24. Umemura, S., D. Marver, D. D. Smyth, and W. A. Pettinger. 1985. $\alpha_{2}$-adrenoceptors and cellular cAMP levels in single nephron segments from the rats. Am. J. Physiol. 249:F28-F33.

25. Umemura, S., D. D. Smyth, and W. A. Pettinger. 1985. Lack of inhibition by atrial natriuretic factor on cyclic AMP levels in single nephron segments and the glomerulus. Biochem. Biophys. Res. Commun. 127:943-949.

26. Inui, K., H. Saito, Y. Matsukawa, K. Nakao, N. Morii, H. Imura, M. Shimokura, Y. Kiso, and R. Hori. 1985. Specific binding activities and cyclic GMP responses by atrial natriuretic polypeptide in kidney epithelial cell line (LLC-PK 1 ). Biochem. Biopys. Res. Commun. 132: 253-260.

27. Manning, P., D. Schwartz, N. C. Katsube, S. W. Holmberg, and P. Needleman. 1985. Vasopressin-stimulated release of atriopeptin: endocrine antagonists in fluid homeostasis. Science (Wash. DC). 229:395397.

28. Tanaka, I., K. S. Misono, and T. Inagami. 1984. Atrial natriuretic factor in rat hypothalamus, atria and plasma: determination by specific radioimmunoassay. Biochem. Biophys. Res. Commun. 124:663-668.

29. Gutkowska, J., K. Horky, G. Thibault, P. Januszewicz, M. Cantin, and J. Genest. 1984. Atrial natriuretic factor is a circulating hormone. Biochem. Biophys. Res. Commun. 125:315-323.

30. Lang, R. E., H. Tholken, D. Ganten, F. C. Luft, H. Ruskoaho, and T. Unger. 1985. Atrial natriuretic factor-a circulating hormone stimulated by volume loading. Nature (Lond.). 314:264-266.

31. Wakitani, K., T. Oshima, A. D. Loewy, S. W. Holmberg, B. R. Cole, S. P. Adams, K. F. Fok, M. G. Currie, and P. Needleman. 1985. Comparative vascular pharmacology of the atriopeptins. Circ. Res. 56: 621-627.
32. Atarashi, K., P. J. Mulrow, and R. Franco-Saenz. 1985. Effect of atrial peptides on aldosterone production. J. Clin. Invest. 76:18071811.

33. Chartier, L., E. Schiffrin, and G. Thibault. 1984. Effect of atrial natriuretic factor-related peptides on aldosterone secretion by adrenal glomerulosa cells: critical role of the intramolecular disulphide bond. Biochem. Biophys. Res. Commun. 122:171-174.

34. Rapoport, R., and F. Murad. 1983. Endothelium-dependent and nitrovasodilator-induced relaxation of vascular smooth muscle: role of cyclic GMP. J. Cyclic Nucleotide Protein Phosphorylation Res. 9:281296.

35. Hughes, J. M., F. Murad, B. Chang, and R. L. Guerrant. 1978. Role of cyclic GMP in the action of heat-stable enterotoxin of Escherichia coli. Nature (Lond.). 271:755-756.

36. Field, M., L. H. Graf, W. J. Laird, and P. L. Smith. 1978. Heatstable enterotoxin of Escherichia coli: in vitro effects on guanylate cyclase activity, cyclic GMP concentration, and ion transport in small intestine. Proc. Natl. Acad. Sci. USA. 75:2800-2804.

37. Guerrant, R., J. M. Hughes, B. Chang, D. C. Robertson, and F. Murad. 1980. Activation of intestinal guanylate cyclase by heat-stable enterotoxine of Escherichia coli: studies of tissue specificity, potential receptors, and intermediates. J. Infect. Dis. 142:220-228.

38. Snider, R. M., M. McKinney, C. Forray, and E. Richelsen. 1984. Neurotransmitter receptors mediate cyclic GMP formation by involvement of arachidonic acid and lipoxygenase. Proc. Natl. Acad. Sci. USA. 81:3905-3909.

39. Davis, C. L., J. Schnermann, and J. Briggs. 1986. Effect of atrial natriuretic factor on medullary solute gradients. Kidney Int. 29:414a. (Abstr.)

40. Bianchi, C., J. Gutkowska, G. Thibault, R. Garcia, and M. Cantin. 1985. Radioautographic localization of ${ }^{125} \mathrm{I}$-atrial natriuretic factor in rat tissues. Histochemistry. 82:441-452.

41. Koseki, C., Y. Hayashi, S. Torikai, M. Furuya, N. Ohnuma, and M. Imai. 1986. Localization of binding for $\alpha$-rat atrial natriuretic polypeptide in rat kidney. Am. J. Physiol. 250:F210-F216.

42. Zeidel, M. L., J. L. Seifter, B. M. Brenner, and P. Silva. 1986. Atrial peptides inhibit oxygen consumption in kidney medullary collecting duct cells. Am. J. Physiol. 251:F379-F383.

43. Werness, J., S. Honma, S. M. Gapstur, and T. P. Dousa. 1986. Inhibitory effect of atrial natriuretic peptide on cAMP accumulation in glomeruli and in cortical collecting tubules microdissected from the rat kidney. Kidney Int. 29:425a. (Abstr.)

44. Umemura, S., D. D. Smyth, and W. A. Pettinger. 1986. $\alpha_{2}$-Adrenoceptor stimulation in microdissected rat glomeruli. Am. J. Physiol. 250:F103-F108. 